\title{
Quarkonium production to explore hadron 3D structure
}

\section{Miguel G. Echevarria*}

INFN, Sezione di Pavia, Via Bassi 6, 27100 Pavia, Italy

E-mail: mgechevarriadpv.infn.it

We discuss how quarkonium production, in particular $\eta_{c}$ production in proton-proton collisions, can be used to access gluon transverse-momentum-dependent parton distribution functions (TMDPDFs). To do so, we apply the effective field theory machinery to factorize the process in terms of gluon TMDPDFs at low transverse momentum, and match this result with the collinear framework to obtain the full transverse-momentum spectrum. This matching is performed by applying the newly devised inverse-error weighting method, based on an estimation of the uncertainties coming from power corrections to construct a weighted average of both factorization theorems.

XVII International Conference on Hadron Spectroscopy and Structure - Hadron2017

25-29 September, 2017

University of Salamanca, Salamanca, Spain

\footnotetext{
* Speaker.
} 


\section{Introduction}

Quarkonium production is an interesting class of processes potentially useful to access gluon TMDPDFs (see e.g. Refs. [1-19]), which encode the rich three-dimensional inner structure of the hadrons in momentum space (see e.g. Ref. [20]). Below we consider one of them: $\eta_{c}$ production in proton-proton collisions at the LHC, for which the hard scale $Q=M_{\eta_{c}}=2.98 \mathrm{GeV}$ [21]. Since the hard scale is so low, there is no a clear separation between the regions in transverse momentum where either the TMD or the collinear frameworks can be applied, which makes this process very sensitive to the applied matching scheme, and which can affect the future extraction of the gluon TMDPDFs from experimental data.

\section{TMD and collinear frameworks}

At low transverse momentum the cross-section is factorized in terms of gluon TMDPDFs [2224] (see also Refs. [25-27]), whereas at high transverse momentum the cross-section is factorized in terms of collinear integrated PDFs. Below we present the relevant results for both frameworks.

The formalism for the production of a pseudo-scalar quarkonioum $\eta_{c}$ at low transverse momentum has been presented in Refs. [3, 14,28]. Following an effective theory approach [14,28,29] based on a combination of soft-collinear effective theory and non-relativistic QCD (NRQCD), and considering unpolarized protons only, we derive the needed TMD factorization theorem.

The most common approach to calculate quarkonium production is the NRQCD formalism [30], where we can write the cross-section for $\eta_{c}$ production in proton-proton collisions as:

$$
d \sigma\left[p p \rightarrow \eta_{c} X\right]=\sum_{n} d \sigma[p p \rightarrow c \bar{c}(n) X]\left\langle\mathscr{O}^{\eta_{c}}(n)\right\rangle
$$

where $d \sigma[p p \rightarrow c \bar{c}(n) X]$ is the short-distance cross-section for producing the $c \bar{c}$ pair in a state $n$ with definite color and angular momentum quantum numbers and $\left\langle\mathscr{O}^{\eta_{c}}(n)\right\rangle$ is a long distance matrix element (LDME) that describes the non-perturbative formation of the bound-state $\eta_{c}$ from the $c \bar{c}$ pair in the state $n . X$ denotes other possible particles in the final state which are integrated over. The quantum numbers $n$ will be denoted by $\left.{ }^{2 S+1} L{ }^{[} i\right]_{J}$, where the notation for angular momentum is standard and $i=1(8)$ for color-singlet (color-octet) states. The short distance cross-sections are perturbatively calculable (apart from the parton distribution functions) in a power series in $\alpha_{s}$, while the LDMEs are nonperturbative and must be extracted from data. The LDMEs scale with definite powers of the quark-pair relative velocity $v$, so the NRQCD factorization formalism organizes the calculation of quarkonium production (and decay) into a systematic double expansion in $\alpha_{s}$ and $v$.

Given that the color singlet state ${ }^{1} S_{0}$ dominates this process, we can argue that it is analogous to Higgs boson production in proton-proton collisions, in the sense that we have a glue-glue fusion 
into a color-singlet state, and then make use of the following TMD factorization ansatz:

$$
\begin{aligned}
\frac{d \sigma}{d y d^{2} q_{T}} & =\frac{2 \pi^{3} \alpha_{s}^{2}}{9 s M_{\eta_{c}}^{3}} H\left(\mu^{2}, M_{\eta_{c}}^{2}\right)\left\langle\mathscr{O}^{\eta_{c}}\left({ }^{1} S_{0}\right)\right\rangle \int \frac{d^{2} b_{T}}{(2 \pi)^{2}} e^{i b_{T} \cdot q_{T}} \\
& \times\left[\tilde{f}_{1 g / A}\left(x_{A}, b_{T} ; \mu, \zeta_{A}\right) \tilde{f}_{1 g / B}\left(x_{B}, b_{T} ; \mu, \zeta_{B}\right)-\tilde{h}_{1 g / A}^{\perp(2)}\left(x_{A}, b_{T} ; \mu, \zeta_{A}\right) \tilde{h}_{1 g / B}^{\perp(2)}\left(x_{B}, b_{T} ; \mu, \zeta_{B}\right)\right] \\
& +\left[\mathscr{O}\left(q_{T} / M_{\eta_{c}}\right)^{a}\right] \sigma .
\end{aligned}
$$

The NRQCD matrix element is [30] $\left\langle\mathscr{O}^{\eta_{c}}\left({ }^{1} S_{0}\right)\right\rangle=\frac{N_{c}}{2 \pi}\left|R_{n}(0)\right|^{2}\left[1+\mathscr{O}\left(v^{4}\right)\right]$, where $R_{n}$ is the radial wave-function of the $q \bar{q}$ pair with quantum number $n$, and $v$ is the relative velocity of the quarks in the pair. According to Ref. [31] (see Table I) we choose $\left|R_{n}(0)\right|^{2}=0.921533 \mathrm{GeV}^{-3}$.

The gluon TMDPDFs $\tilde{f}_{1}^{g}$ and $\tilde{h}_{1}^{\perp g(2)}$ provide the distribution of the initial state gluons [22-24] as a function of their collinear momentum fraction $x_{h}$, at given values for the UV-renormalization and rapidity-renormalization scales (e.g. $\mu^{2}=\zeta_{h}=M_{\eta_{c}}^{2}$ ). The function $\tilde{f}_{1}^{g}\left(x, k_{T}\right)$ is the TMDPDF for unpolarized gluons in unpolarized hadrons whereas the $\tilde{h}_{1}^{\perp g(2)}\left(x, k_{T}\right)$ TMDPDF accounts for linearly polarized gluons in unpolarized protons [23]. The Fourier transforms of the functions and their moments $\tilde{f}_{1}^{g}\left(x, b_{T}\right), \tilde{h}_{1}^{\perp g(2)}\left(x, b_{T}\right)$ are defined in [23].

A necessary condition for the factorization theorem to hold is that the structure of the infrared poles at a specific perturbative order in $\alpha_{s}$ is the same for the cross-section in full QCD and in the factorized form (the hard part $H$ should be free from infrared divergences) This argument can be used backwards, to establish a factorization theorem ansatz at a given perturbative order by checking if the obtained hard part (by subtraction) is actually free from divergences. For $\eta_{q}$ production this has been verified at $\mathscr{O}\left(\alpha_{s}\right)[28,29]$, where the hard part $H$ is ${ }^{1}$

$$
H^{(1)}=\sigma_{\mathrm{virt}}^{(1)}-\left[\tilde{f}_{1}^{g / A} \tilde{f}_{1}^{g / B}\right]_{\mathrm{virt}}^{(1)}=\frac{\alpha_{s}}{2 \pi}\left[-C_{A} \ln ^{2} \frac{\mu^{2}}{M_{\eta_{c}}^{2}}+2 C_{A}\left(1+\frac{\pi^{2}}{3}\right)+2 C_{F}\left(-5+\frac{\pi^{2}}{4}\right)\right]
$$

with $H^{(0)}=1$. This result, together with the known gluon TMDPDFs, allows us to perform the resummation of large logarithms at NNLL accuracy.

At large transverse momentum $\left(q_{T} \sim M_{\eta_{c}} \gg m \sim 1 \mathrm{GeV}\right)$ the cross-section is described by collinear factorization. For the unpolarized case, consistently with the $\alpha_{s}$ accuracy at low $q_{T}$, we describe the cross-section at fixed $\mathscr{O}\left(\alpha_{s}^{3}\right)$ order. At $q_{T} \geq M_{\eta_{c}}$ the hard scale is given by the transverse mass $m_{T}=\sqrt{M_{\eta_{c}}^{2}+q_{T}^{2}}$ and the cross-section is given by [32] ${ }^{2}$

$$
\begin{aligned}
\frac{d \sigma}{d y d^{2} q_{T}} & =\sum_{a, b} \int d x_{a} d x_{b} f_{1}^{a / A}\left(x_{a} ; \mu\right) f_{1}^{b / B}\left(x_{b} ; \mu\right) \delta\left(\hat{s}+\hat{t}+\hat{u}-M_{h}^{2}\right) \frac{\hat{s}}{\pi} \frac{d \sigma}{d \hat{t}}(a b \rightarrow h d) \\
& +\left[\mathscr{O}\left(m / q_{T}\right)^{b}\right] \sigma,
\end{aligned}
$$

where $a, b$ are partons in the initial state, $h$ is the produced hadron and $d$ a parton radiated in the final state. $\hat{s}, \hat{t}, \hat{u}$ are the partonic Mandelstram variables [33] and the partonic cross-section $d \sigma / d \hat{t}$ is given at $\mathscr{O}\left(\alpha_{s}^{3}\right)$ in Refs. [34-36] for different channels.

\footnotetext{
${ }^{1}$ We notice that only the virtual contributions are necessary to obtain the hard part of the TMD factorization theorem, since real-gluon emission diagrams live at a lower scale and match exactly between the full and the factorized theories.

${ }^{2}$ Note that we generalize the result in [32] to the massive case.
} 

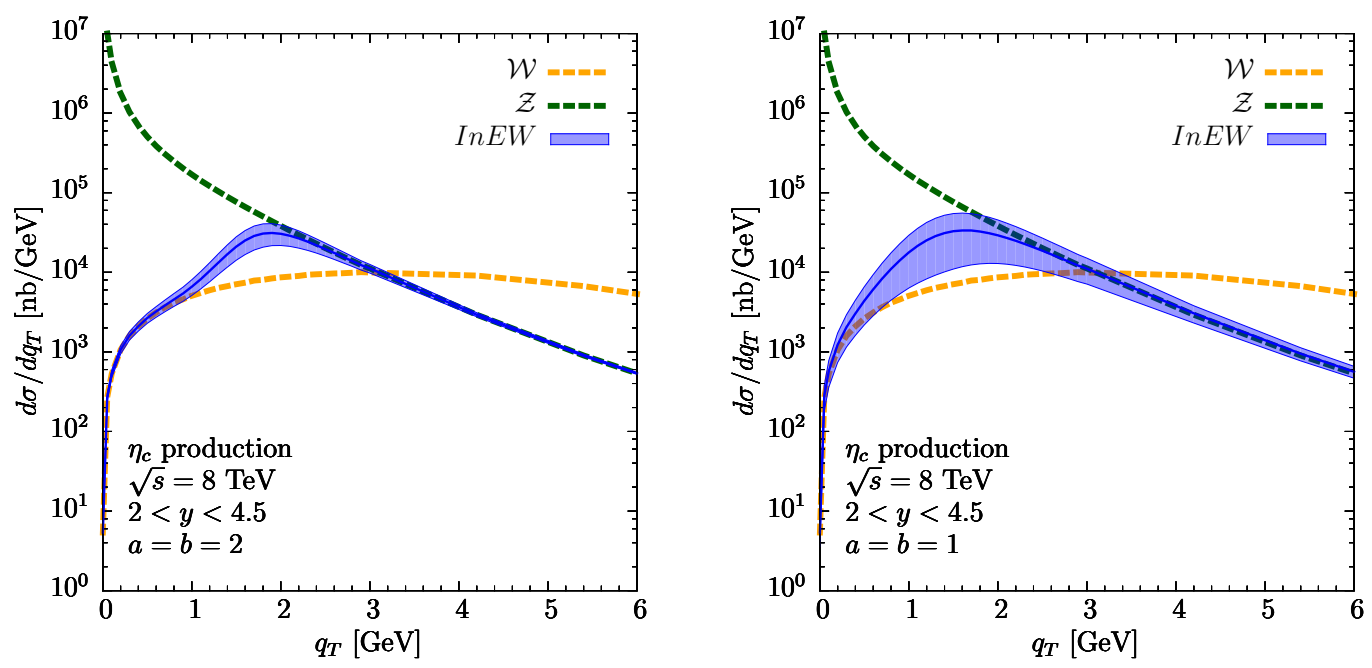

Figure 1: The TMD resummed term $\mathscr{W}$ (yellow curve), the collinear fixed-order term $\mathscr{Z}$ (green curve), and the matched cross section with the inverse-error weighting ( $I n E W)$ method (blue band) for $\eta_{c}$ production in proton-proton collisions at $\sqrt{s}=8 \mathrm{TeV}$.

\section{Phenomenology: the complete transverse-momentum spectrum}

In Fig. 1 we show the matched cross-section for $\eta_{c}$ production in proton-proton collisions at the LHC at $\sqrt{s}=8 \mathrm{TeV}$. To do so, we apply the recently devised inverse-error weighting method $(I n E W)$ [37], which makes use of estimations of the power-corrections to both TMD and collinear factorization theorems to construct their weighted average. As can be seen, the matched crosssection overlaps with the TMD result ( $\mathscr{W})$ at low $q_{T}$ and with the collinear result ( $\mathscr{Z}$ ) at large $q_{T}$, as expected. The values of $a$ and $b$ are related to the strength of the power corrections, and varied here between 1 and 2 to show their impact. Notice that the uncertainty on the matched cross section is only due to the matching scheme, i.e. including power-correction uncertainties, and no other effects are added, such as the perturbative-scale variations and the non-perturbative contributions. All these effects should be properly included in an actual comparison to experimental data.

\section{Acknowledgements}

This work was supported by the European Research Council (ERC) under the European Union's Horizon 2020 research and innovation program (grant agreement No. 647981, 3DSPIN).

\section{References}

[1] J.-W. Qiu, M. Schlegel and W. Vogelsang, Probing Gluonic Spin-Orbit Correlations in Photon Pair Production, Phys. Rev. Lett. 107 (2011) 062001, [1103.3861].

[2] D. Boer, W. J. den Dunnen, C. Pisano, M. Schlegel and W. Vogelsang, Linearly Polarized Gluons and the Higgs Transverse Momentum Distribution, Phys. Rev. Lett. 108 (2012) 032002, [1109. 144 4].

[3] D. Boer and C. Pisano, Polarized gluon studies with charmonium and bottomonium at LHCb and AFTER, Phys. Rev. D86 (2012) 094007, [1208.3642]. 
[4] P. Sun, C. P. Yuan and F. Yuan, Heavy Quarkonium Production at Low Pt in NRQCD with Soft Gluon Resummation, Phys. Rev. D88 (2013) 054008, [1210.3432].

[5] J. P. Ma, J. X. Wang and S. Zhao, Transverse momentum dependent factorization for quarkonium production at low transverse momentum, Phys. Rev. D88 (2013) 014027, [1211. 7144 ].

[6] R. Zhu, P. Sun and F. Yuan, Low Transverse Momentum Heavy Quark Pair Production to Probe Gluon Tomography, Phys. Lett. B727 (2013) 474-479, [1309.0780].

[7] C. Pisano, D. Boer, S. J. Brodsky, M. G. A. Buffing and P. J. Mulders, Linear polarization of gluons and photons in unpolarized collider experiments, JHEP 10 (2013) 024, [1307 . 3417 ].

[8] W. J. den Dunnen, J. P. Lansberg, C. Pisano and M. Schlegel, Accessing the Transverse Dynamics and Polarization of Gluons inside the Proton at the LHC, Phys. Rev. Lett. 112 (2014) 212001, [1401.7611].

[9] G.-P. Zhang, Transverse momentum dependent gluon fragmentation functions from $J / \psi \pi$ production at $e^{+} e^{-}$colliders, Eur. Phys. J. C75 (2015) 503, [1504.06699].

[10] D. Boer and C. Pisano, Impact of gluon polarization on Higgs boson plus jet production at the LHC, Phys. Rev. D91 (2015) 074024, [1412.5556].

[11] J. P. Ma and C. Wang, QCD factorization for quarkonium production in hadron collisions at low transverse momentum, Phys. Rev. D93 (2016) 014025, [1509. 04421 ].

[12] D. Boer, P. J. Mulders, C. Pisano and J. Zhou, Asymmetries in Heavy Quark Pair and Dijet Production at an EIC, JHEP 08 (2016) 001, [1605.07934].

[13] A. Mukherjee and S. Rajesh, Linearly polarized gluons in charmonium and bottomonium production in color octet model, Phys. Rev. D95 (2017) 034039, [1611.05974].

[14] A. Signori, Gluon TMDs in quarkonium production, Few Body Syst. 57 (2016) 651-655, [1602.03405].

[15] J.-P. Lansberg, C. Pisano and M. Schlegel, Associated production of a dilepton and $a \Upsilon(J / \psi)$ at the LHC as a probe of gluon transverse momentum dependent distributions, Nucl. Phys. $\mathbf{B 9 2 0}$ (2017) 192-210, [1702.00305].

[16] A. V. Efremov, N. Ya. Ivanov and O. V. Teryaev, QCD predictions for the azimuthal asymmetry in charm leptoproduction for the COMPASS kinematics, Phys. Lett. B772 (2017) 283-289, [1706.03125].

[17] R. M. Godbole, A. Kaushik and A. Misra, Transverse single-spin asymmetry in the low-virtuality leptoproduction of open charm as a probe of the gluon Sivers function, 1709.03074.

[18] G.-P. Zhang, Back-to-back heavy quark pair production in Semi-inclusive DIS, JHEP 11 (2017) 069, [1709.08970].

[19] J.-P. Lansberg, C. Pisano, F. Scarpa and M. Schlegel, Pinning down the linearly-polarized gluons inside unpolarized protons using quarkonium-pair production at the LHC, 1710.01684.

[20] R. Angeles-Martinez et al., Transverse Momentum Dependent (TMD) parton distribution functions: status and prospects, Acta Phys. Polon. B46 (2015) 2501-2534, [1507. 05267].

[21] Particle Data Group collaboration, C. Patrignani et al., Review of Particle Physics, Chin. Phys. C40 (2016) 100001.

[22] P. J. Mulders and J. Rodrigues, Transverse momentum dependence in gluon distribution and fragmentation functions, Phys. Rev. D63 (2001) 094021, [hep-ph/ 000934 3]. 
[23] D. Boer, S. Cotogno, T. van Daal, P. J. Mulders, A. Signori and Y.-J. Zhou, Gluon and Wilson loop TMDs for hadrons of spin $\leq$ 1, JHEP 10 (2016) 013, [1607.01654].

[24] M. G. Echevarria, T. Kasemets, P. J. Mulders and C. Pisano, QCD evolution of (un)polarized gluon TMDPDFs and the Higgs $q_{T}$-distribution, JHEP 07 (2015) 158, [1502. 05354 ].

[25] J. Collins, Foundations of perturbative QCD. Cambridge University Press, 2013.

[26] M. G. Echevarria, A. Idilbi and I. Scimemi, Factorization Theorem For Drell-Yan At Low $q_{T}$ And Transverse Momentum Distributions On-The-Light-Cone, JHEP 07 (2012) 002, [1111. 4996 ].

[27] M. G. Echevarria, A. Idilbi and I. Scimemi, Soft and Collinear Factorization and Transverse Momentum Dependent Parton Distribution Functions, Phys. Lett. B726 (2013) 795-801, [1211.1947].

[28] A. Signori, Flavor and Evolution Effects in TMD Phenomenology, Ph.D. thesis, Vrije U., Amsterdam, 2016.

[29] M. G. Echevarria, T. Kasemets, J.-P. Lansberg, C. Pisano and A. Signori. In preparation.

[30] G. T. Bodwin, E. Braaten and G. P. Lepage, Rigorous QCD analysis of inclusive annihilation and production of heavy quarkonium, Phys. Rev. D51 (1995) 1125-1171, [hep-ph/9407339].

[31] M. Butenschoen, Z.-G. He and B. A. Kniehl, $\eta_{c}$ production at the LHC challenges nonrelativistic-QCD factorization, Phys. Rev. Lett. 114 (2015) 092004, [1411. 5287].

[32] CTEQ collaboration, R. Brock et al., Handbook of perturbative QCD: Version 1.0, Rev. Mod. Phys. 67 (1995) 157-248.

[33] M. E. Peskin and D. V. Schroeder, An Introduction to quantum field theory. Addison-Wesley, Reading, USA, 1995.

[34] R. Baier and R. Ruckl, Hadronic Collisions: A Quarkonium Factory, Z. Phys. C19 (1983) 251.

[35] J. H. Kuhn and E. Mirkes, QCD corrections to toponium production at hadron colliders, Phys. Rev. D48 (1993) 179-189, [hep-ph/9301204].

[36] A. Petrelli, M. Cacciari, M. Greco, F. Maltoni and M. L. Mangano, NLO production and decay of quarkonium, Nucl. Phys. B514 (1998) 245-309, [hep-ph/ 9707223 ].

[37] M. G. Echevarria, T. Kasemets, J.-P. Lansberg, C. Pisano and A. Signori, Matching factorization theorems with an inverse-error weighting, 1801.01480. 